\title{
Concurrent Support of Important Buses using Placing Minimal Number of Phasor Measurement Components
}

\author{
Rajesh Kumar Samala ${ }^{1}$, A Santosh Kumar ${ }^{2}$, V. Kamaraju ${ }^{3}$ \\ ${ }^{\prime}$ (Assistant Professor, Department of Electrical Engineering, Nalla Narasimha Reddy Education Society's \\ Group of Institutions, Hyderabad, India ) \\ ${ }^{2}$ (M.Tech Scholar, Department of Electrical Engineering, Sreenidhi Institute of Science \& Technology, \\ Hyderabad, India ) \\ ${ }^{3}$ (Professor, Department of Electrical Engineering, Mahaveer Institute of Science \& Technology, Hyderabad, \\ India)
}

\begin{abstract}
A mode of procedure for arrangement of Phasor Measurement Components (PMC), make sure that the support of injured buses which are achieved based on transient and voltage stability analysis process on the whole system is projected. Arrangement of PMCs can be done based on various criterions. Topological observability is one of the significant prospect that has been directed in the past thereby empower the Wide Area Support to be run totally independent from SCADA stage. The arrangement is prejudiced by the following criteria- less number of substations, less number of PMC components, observability of the topology based on the PMCs, in view of the PMC type. Concurrent support of phase angles and voltage stability indices crossways different nodes point toward the closeness to instability. The matter is to identify the injured buses where the PMCs should be positioned when the transient stability and voltage calculation is taken into report. Integer Linear Programming with equality and inequality constraints is used to detect the most excellent arrangement set with injured buses recognized from transient stability analysis. The projected mode of procedure has been examined on Indian Southern Grid 24 modified bus system in detail and the results have been included.
\end{abstract}

Keywords: Integer Linear Programming, Phasor Measurement Components, Phase angles, Transient stability and Voltage stability

\section{Introduction}

Current phasor measurement systems can find out their inauguration to the progress of the Symmetrical Component Distance Relay (SCDR) in the early 1971s.[1] The microcomputers of that stage were not having capacity of managing the necessities of a distance relay program. This lead to the development of the Symmetrical Component Distance Relay (SCDR) which is utilized symmetrical components of voltages and currents in order to change the 6 fault equations of a three phase transmission line into a single mathematical equation utilizing symmetrical components. After a while it became very clear that the microcomputers had become adequately capable so that this development was no longer essential for line relaying. On the other hand, the actuality is that the SCDR used resourceful mode of procedures of calculating symmetrical components of voltages and currents evaluated to be extremely fascinating for other applications. In actual fact, positive sequence component of voltages and currents of a network are the backbone of most power system analysis schedules: load flow, stability, short circuit, most favorable power flow, state estimation, incident analysis, etc.

Coordinated, actual time, phase angle, phasor measurements over a distributed power network currents an outstanding prospect for most important developments in power system control and protection. Coordinated phasor measurements have become a realistic arrangement only for the duration of the last decade particularly with the beginning of fast action microcontrollers and extremely truthful coordination by means of Global Arrangement System (GAS) satellites. The improvement of Phasor Measurement Components (PMC) derived from GPS establishes that it is achievable to monitor system function condition straightly. [2] For it, their prospective for use in power system applications has yet to be fully actualized.

This paper is organized as follows: In the first section the Introduction Concurrent Support of Important Buses using Placing Minimal Number of Phasor Measurement Components is discussed, in the second section Basic definitions and calculations are presented and in the third section credentials of injured buses based on voltage stability analysis are presented, in fourth section integer linear programming is explained and in fifth section conclusion is presented. 


\subsection{Definition and Calculation}

\section{Phasor} waveforms.

A phasor is a composite number that illustration of together the amplitude and phase angles of sinusoidal

\subsection{Conventional Definition of a phasor}

Phasor is a measure with amplitude and phase angles that is utilized to express a sinusoidal signal representation as shown in Fig. 1. At this time the phase angle is the distance between the signals sinusoidal peak and a particular reference and is communicate using an angular measurement. Now, the reference is affixed point in time (reference as time $=0$ ). The phasor amplitude is associated to the amplitude of the sinusoidal waveform signal.

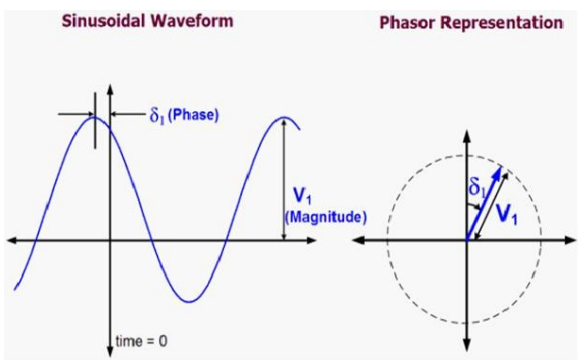

Figure.1 Phasor representation of a sinusoidal signal

The most normally used schedule of calculating phasors from sampled data is that of Discrete Fourier Transform (DFT). The sampling clocks are generally kept at a steady frequency even though the input signal frequency may vary by a little quantity more or less its nominal value.

Phasor measurements that take place at the simultaneously are called "synchrophasors" and can be measured accurately from components called Phasor Measurement Components (PMCs). PMC measurements are taken at high speed usually 35 or 60 observations per second analyze to one every 5 to 11 seconds utilizing conventional knowledge. Every measurement is time stamped according to a regular time references. Time stamping provides synchrophasors from different places to be time associated (or "coordinated") given that mutual and wide-ranging observation of the complete grid. Synchrophasors facilitate an improved suggestion of network stress, and can be utilized to trigger counteractive measures to keep up reliability representation as shown in Fig. 2.

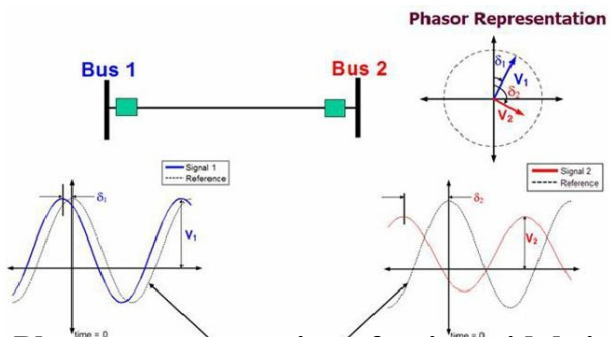

Figure. 2 Phasor representation of a sinusoidal signal.

\subsection{Phasor Measurement Component}

A practical block diagram of a PMC is represents in Figure. 3. The GPS receiver 1 pps signal is utilized in a phase locked loop to generate the sampling clock signals, later they are then utilized for sampling analog signals. Phasors of phase voltages and currents are considered by the recursive DFT algorithms. The calculated phasors are mutual to form positive sequence components, and time tagged with the timing information furnished by the clock and the Second of Century (SOC) count furnished by the GPS receiver.

Phasor knowledge is measured to be one of the best important measurement methods in the scheme of upcoming power systems due to its distinctive capability to sample analog voltage and current waveform data in synchronism with a GPS clock and work out the respective $50 \mathrm{~Hz}$ phasor factor (i.e. composite numbers showing the amplitude and phase angles of a $50 \mathrm{~Hz}$ sinusoidal waveform signal) from extensively dispersed places. This corresponding sampling development of the diverse waveforms furnishes a regular reference for the phasor calculations at all the diverse places. 


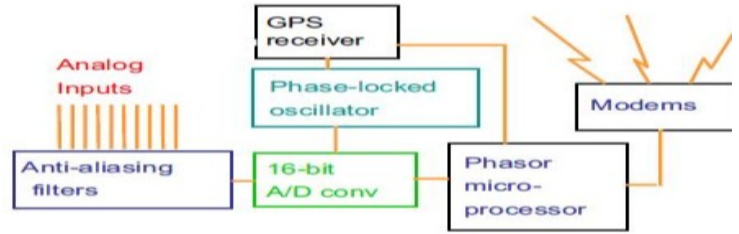

Figure. 3 Block diagram of the elements in a Phasor Measurement Component

\subsection{Voltage stability index $(\mathrm{L})$}

\section{Credentials Of Injured Buses Based On Voltage Stability Analysis}

Let us consider a system where $\mathrm{m}$ is the total number of buses with $1,2,3.4 ., 2 \mathrm{~m}, \mathrm{n}$ number of generator buses, and $\mathrm{n}+1, \ldots, \mathrm{m}$, and the remaining $(\mathrm{m}-\mathrm{n})$ buses are load buses. For a specified system we can write it in the matrix form as,

$$
\left[\begin{array}{c}
I_{G} \\
I_{L}
\end{array}\right]=\left[\begin{array}{cc}
Y_{G G} & Y_{G L} \\
Y_{L G} & Y_{L L}
\end{array}\right]\left[\begin{array}{c}
V_{G} \\
V_{L}
\end{array}\right]
$$

Where $\mathrm{I}_{\mathrm{G}}, \mathrm{I}_{\mathrm{L}}$ and $\mathrm{V}_{\mathrm{G}}, \mathrm{V}_{\mathrm{L}}$ representing composite current and voltage vectors at the generator and load buses. $\left[\mathrm{Y}_{\mathrm{GG}}\right],\left[\mathrm{Y}_{\mathrm{GL}}\right],\left[\mathrm{Y}_{\mathrm{LL}}\right]$ and $\left[\mathrm{Y}_{\mathrm{LG}}\right]$ are corresponding partitioned portions of network $\mathrm{Y}$-bus matrix:

$$
\begin{aligned}
& {\left[\mathrm{I}_{\mathrm{G}}\right]=\left[\mathrm{Y}_{\mathrm{GG}}\right]\left[\mathrm{V}_{\mathrm{G}}\right]+\left[\mathrm{Y}_{\mathrm{GL}}\right]\left[\mathrm{V}_{\mathrm{L}}\right]} \\
& {\left[\mathrm{I}_{\mathrm{L}}\right]=\left[\mathrm{Y}_{\mathrm{LG}}\right]\left[\mathrm{V}_{\mathrm{G}}\right]+\left[\mathrm{Y}_{\mathrm{LL}}\right]\left[\mathrm{V}_{\mathrm{L}}\right]}
\end{aligned}
$$

From Equation. (3)

$$
\left[Y_{L L}\right]^{-1}\left[I_{L}\right]=\left[Y_{L L}\right]^{-1}\left[Y_{L G}\right]\left[V_{G}\right]+\left[V_{L}\right]
$$

Substituting $\left[\mathrm{V}_{\mathrm{L}}\right]$ in Equation (2).

$$
\left[V_{L}\right]=\left[Y_{L L}\right]^{-1}\left[I_{L}\right]-\left[Y_{L L}\right]^{-1}\left[Y_{L G}\right]\left[V_{G}\right]
$$

$$
\left[I_{G}\right]=\left[Y_{G G}\right]\left[V_{G}\right]+\left[Y_{G L}\right]\left\{\left[Y_{L L}\right]^{-1}\left[I_{L}\right]-\left[Y_{L L}\right]^{-1}\left[Y_{L G}\right]\left[V_{G}\right]\right\}
$$

Representing Equations. (4) and (5) in matrix form we get,

$$
\left[\begin{array}{c}
V_{L} \\
I_{G}
\end{array}\right]=\left[\begin{array}{cc}
Z_{L L} & F_{L G} \\
K_{G L} & Y_{G G}
\end{array}\right]\left[\begin{array}{c}
I_{L} \\
V_{G}
\end{array}\right]
$$

where $\left[F_{L G}\right]=-\left[Y_{L L}\right]^{-1}\left[Y_{L G}\right]$.

The elements of $\left[\mathrm{F}_{\mathrm{LG}}\right]$ matrix are composite and its columns correspond to the generator bus numbers and rows correspond to the load bus numbers. This matrix provides the relationship between load bus voltages and generator bus voltages. It also provides information about the position of load nodes with respect to generator nodes that is named as relative electrical distance between load nodes and generator nodes.

For a provided system operating situation, utilizing the load flow (state estimation) outcomes, the voltage stability index, $\mathrm{L}$, is calculated as represented in equation (7).

$$
L_{j}=\left|1-\sum_{i=1}^{i=g} F_{j i} \frac{V_{i}}{V_{j}}\right|
$$

The value of L-index lies between 0 and 1 . An L-index value less than 1 (componenty) and close to 0 (zero) represents an enhanced voltage stability boundary. The values $\mathrm{F}_{\mathrm{ji}}$ are achieved from the Y-bus matrix provided by representing in the equation (6).

\subsection{Projected Approach}

The projected approaches for determining the injured buses comprises of reading the data from the buses, transmission lines, etc and choose a transmission line on which the incident analysis is to be carried out and achieve the exhaustive voltage stability analysis on all 1 lines and maximum $L$ index value is found out. Merit list the buses in downward order and classify them into groups to determine the important buses. 


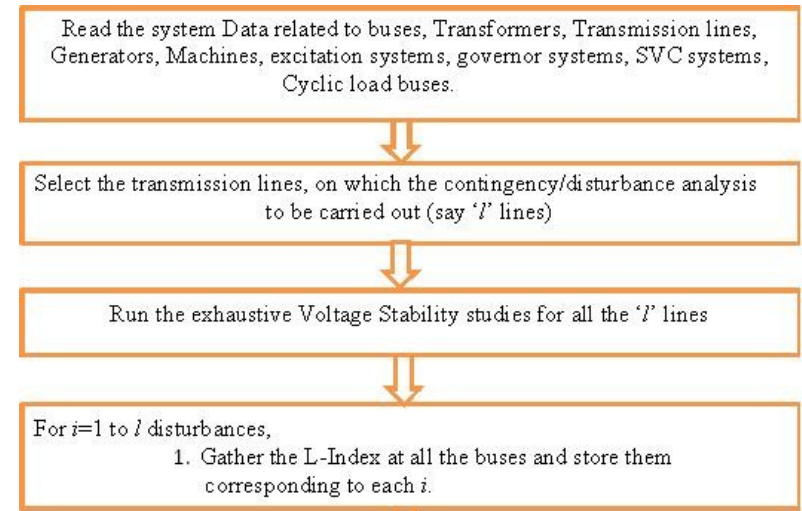

Figure 4: Algorithm for the projected approach

Carry out observ ability analysis using these vulnerable buses by using an optimization method.

The Fig. 5 showing the single line diagram of 24-bus corresponding EHV system. It consists of four generator buses and eight load buses. The generators are located at buses 1, 2, 3 and 4 respectively and the load buses are located at buses $5,6,7,8,9,10,13$ and 15 respectively.

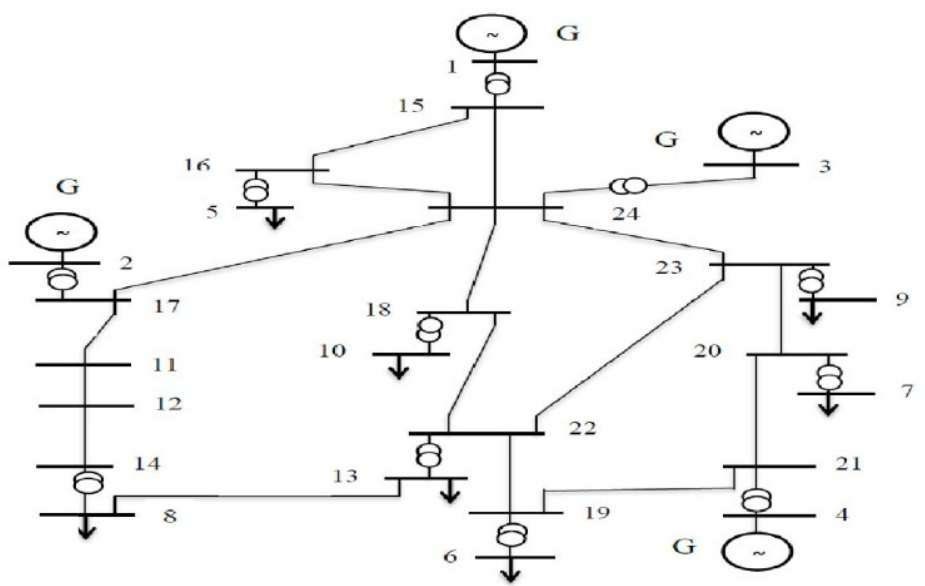

Figure 5: Single line diagram of 24-bus corresponding of Indian Southern Grid

Fig. 6 provides the values of L-index of the 24 bus system in the reference case (100 percent loading at all the buses).

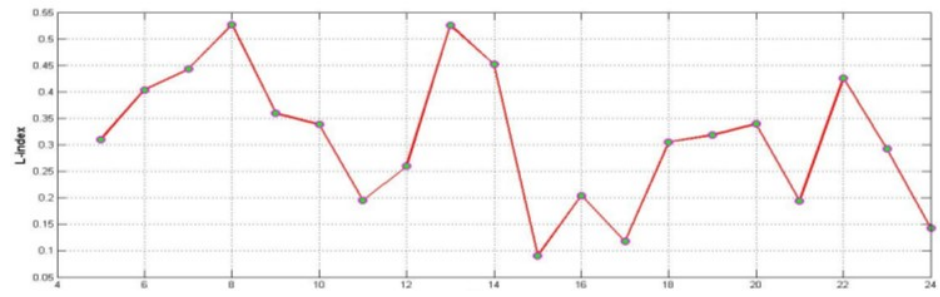

Figure 6: L-index for 24-bus system with 100 percent loading

Fig. 7 represents the L-index value for a 10 percent increase of load at Bus No.5.

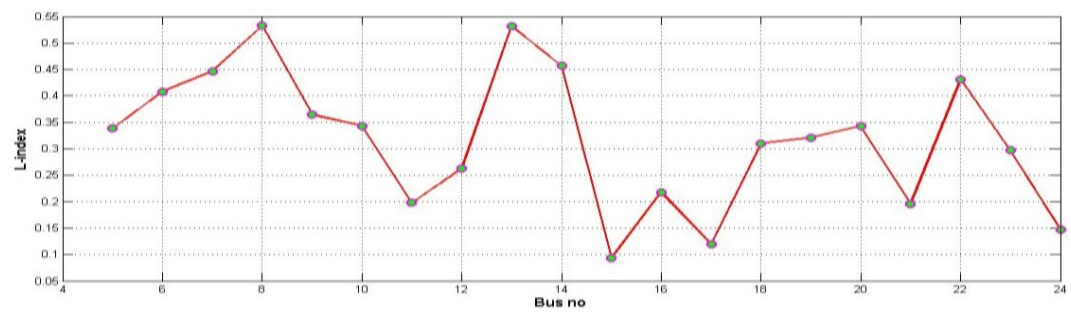

Figure 7: L-index for 24-bus system with 110 percent loading at Bus No.5. 
Fig. 8 represents the L-index values for 10 percent of load increment at Bus No.6.

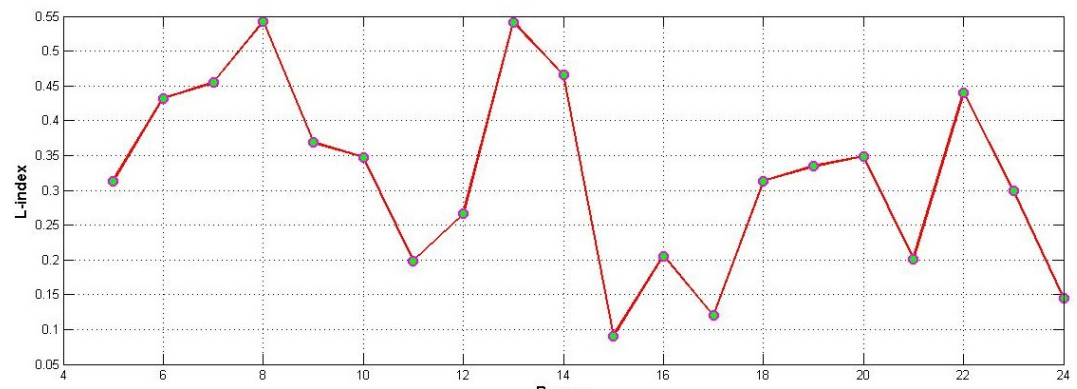

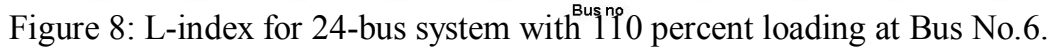

L-index values for 10 percent load increment at Bus Numbers 7 and 8 are represents in Figure. 9 and Figure 10 respectively.

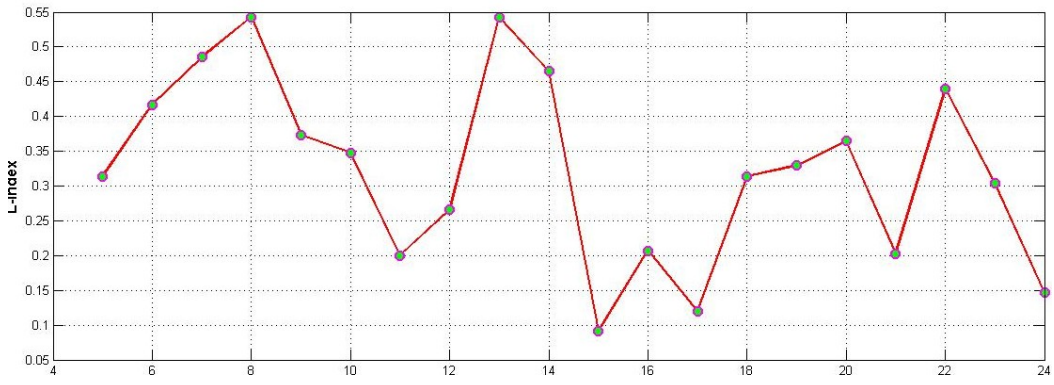

Figure 9: L-index for 24-bus system with 110 percent loading at Bus No.7.

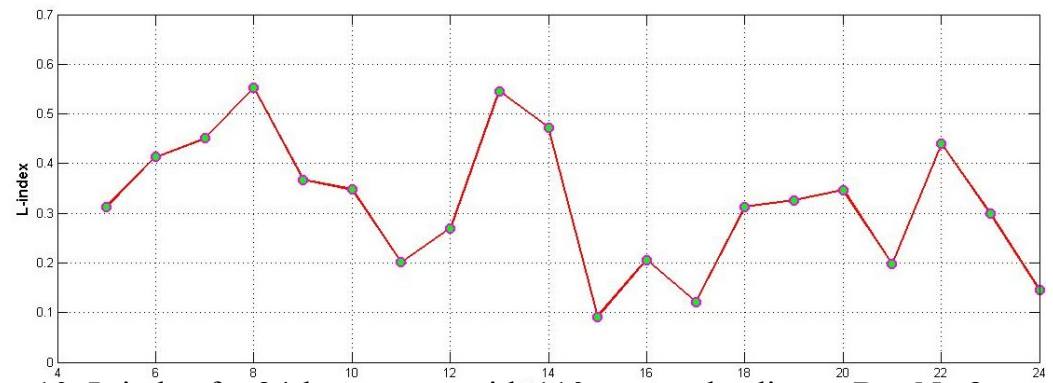

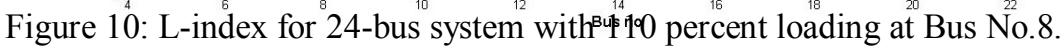

\subsection{Classification of buses}

24-bus corresponding of Indian Southern Grid. For every increment of 10 percent load at different load buses, buses are structured in the downward order based on the value of L-index. Table 1 represents the bus number and corresponding number of times each bus appears in each group. The bus that appears more number of times in the group is considered as the very important bus.

Table 1: L-Index of 24 Bus Systems

\begin{tabular}{|c|l|l|l|l|}
\hline Bus no & st & nd & rd & th \\
\hline 14 & $10 / 10$ & - & - & - \\
\hline 22 & - & $10 / 10$ & - & - \\
\hline 20 & - & - & $10 / 10$ & - \\
\hline 19 & - & - & - & $10 / 10$ \\
\hline
\end{tabular}

For that reason for the 24-bus corresponding of Indian Southern Grid the majority of injured buses are $14,22,20,19,18,23,16,11,21,24,17$ and 15 respectively in the decreasing order of weakness. 


\section{Credentials of injured buses based on Transient stability analysis}

Stability is one of the factors that play an important role in present power system process. Hazard of instability governs most prospects like system loading limits, operating speeds of the primary and back up protection systems, etc. The phase angle differences across various nodes are measure of static stress across the grid and its closeness to instability. Actual time support of phase angle differences across various nodes is a condition for applying counteractive dealings for system stabilization in large power systems. A wide area measurement technology based on the utilization of coordinated measurement knowledge leads to better efficient calculation of instabilities which might cause continuous outages and potentially large scale blackouts. Transient stability examination can be utilized to determine the angle differences at all the buses in a system for a disturbance taking place in the system. The estimation of the constancy of a power system during a transient requires that the dynamics of the subsystems be decomposed into relative and collective motions. This arrangement must establish a difference between synchronous and frequency stability. This type of arrangement is made possible by representing a system center of angle which is defined as the inertia weighted average of all rotor angles.

$$
\delta_{C O A}=\frac{\sum_{i=1}^{N} \delta_{i} H_{i}}{\sum_{i=1}^{N} H_{i}}
$$

Where $\delta_{\mathrm{i}}$ is the internal machine rotor is angle and $\mathrm{H}_{\mathrm{i}}$ is the respective generator inertia time constant and $\mathrm{N}$ is the number of generators in the system.

\subsection{Case Study-1:24 bus system}

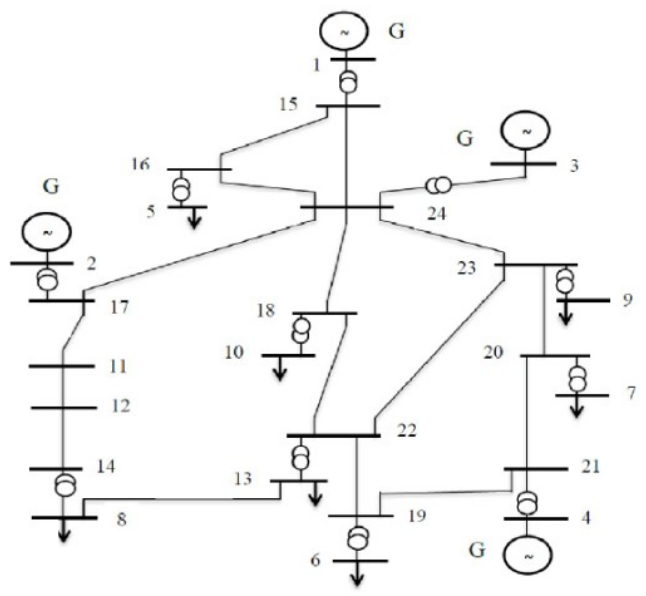

Figure 11: single-line diagram of 24-bus corresponding of Indian Southern Grid

The above Figure.11 shows a single-line diagram of 24-bus corresponding EHV system. It includes four generator buses and eight load buses. The generators are located at bus numbers 1,2,3 and 4 respectively and the load buses are located at bus numbers $5,6,7,8,9,10,13$ and 15 respectively. The disturbance are generated on all the lines and for a disturbance on line $22-18$ is represents as shown Fig. 12.

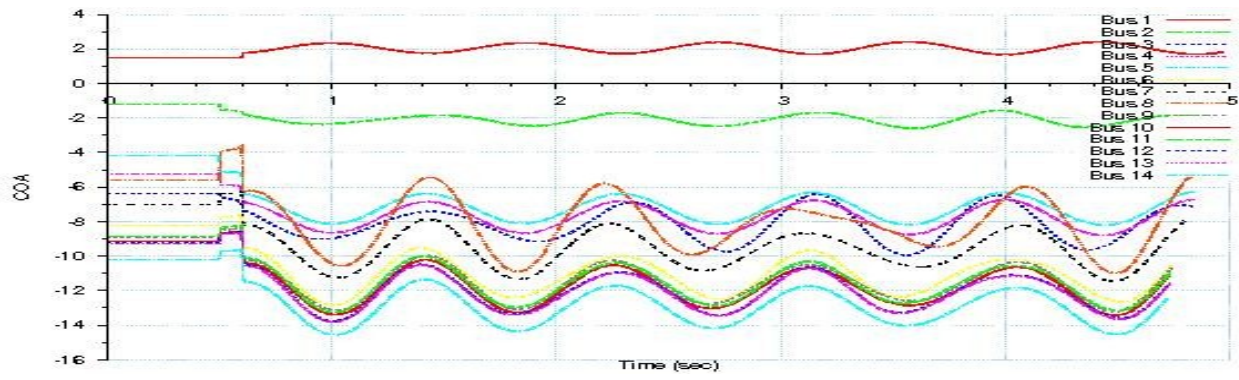

Figure 12: Angular deviations of different buses obtained from transient stability analysis for a line disturbance on line $22-18$ 


\section{Integer Linear Programming (Ilp) Formulation}

Let $x_{i}$ be a binary decision variable related with the bus $i$. Variable $x_{i}$ is set to one if a PMC is placed at bus $i$, otherwise it is set to zero. Then minimum PMC arrangement problem for the given system can be calculated as follows:

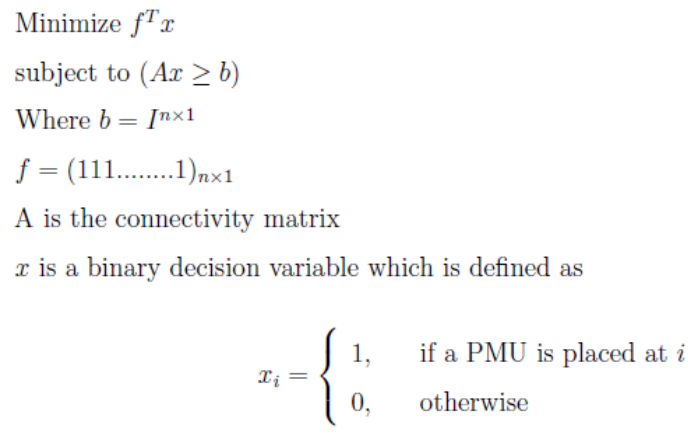

PMC.

Now we will explain the optimization problem for determining the finest places for arrangement of

24-Bus corresponding Indian Southern Grid for this case, with the modified calculations in optimization problem the total number of PMCs needed is 11 for all the cases.

\begin{tabular}{|c|c|c|c|}
\hline No of value & Bus no & Number of PMUs & Location \\
\hline 1 & 14 & 11 & $14,15,16,17,18,19,20,21,22,23,24$ \\
\hline 2 & 14,22 & 11 & $14,15,16,17,18,19,20,21,22,23,24$ \\
\hline 3 & $14,22,22$ & 11 & $14,15,16,17,18,19,20,21,22,23,24$ \\
\hline 4 & $14,22,20,19$ & 11 & $14,15,16,17,18,19,20,21,22,23,24$ \\
\hline
\end{tabular}

\section{Conclusion}

A mode of procedure has been projected to determine injured buses based on voltage stability analysis and it has been implemented on Indian Southern Grid 24 bus system. An Integer Linear Programming (ILP) performance has been formulated to uncover to place the minimal number of PMCs for total observability of power system network. It has been implemented on all the sample test systems considered. A mode of procedure for finest arrangement of Phasor Measurement Components, ensuring the important nodes of a system being support, has been projected. Integer Linear Programming (ILP) is utilized for solving the framed optimization problem.

\section{References}

[1] G. Phadke, M. Ibrahim, T. Hlibka, "Fundamental Basis for Distance Relaying with Symmetrical Components", IEEE Trans. on PAS, Vol. PAS-96, No. 2, March/April 1977, pp. 635-646.

[2] A. G. Phadke, Synchronised phasor measurements in power systems, IEEE Comput. Applicat. Power, vol.6, no. 2, pp. 1015, Apr. 1993.

[3] T. L. Baldwin, L.Mili, M. B. Boisen, Jr. and R. Adapa, Power system observabil-ity with minimal Phasor measurement arrangement, IEEE Transactions on power systems, vol. 8, no.2, May 1993, pp.707-715.

[4] Bei Xu and Ali Abur, Observability analysis and measurement arrangement for systems with PMCs, in Proc. 2004 IEEE PES conf. and Expo., Oct. 10-13, 2004, vol.2, pp.943-946.

[5] Devesh Dua, Sanjay Dambhare, Rajeev Kumar Gajbhiye, and S.A.Soman, Finest Multistage scheduling of PMC arrangement: An ILP approach, IEEE Transactions on power delivery, vol. 23, no. 4, Oct., 2008, pp.1812-1820

[6] Saikat Chakrabarti, Elias Kyriakides, Demetrios G. Eliades, Arrangement of synchro-nized measurements for power system observability, IEEE transactions on power delivery, vol. 24, no. 1, Jan. 2009, pp.12-19.

[7] Ranjana Sodhi, S. C. Srivastava, Finest PMC arrangement to ensure observability of power system, Fifteenth National Power Systems Conference(NPSC), Dec. 2008.

[8] C. Taylor, D. Erickson, B. Wilson, and K. Martin. (2002, December 16). Wide-Area Stability and Voltage Control System (WACS) Demonstration.

[9] U.S.Canada Power System Outage Task Force, Causes of the August 14th Blackout in the Componented States and Canada, Interim Rep., Nov. 2003, Ch 6.

[10] Final report on the August 14, 2003 blackout in the Componented States and Canada: Causes and recommendations, USCanada Power System Outage Task Force, 2004.

[11] C. W. Taylor and D. C. Erickson, Recording and analyzing the July 2 cascading outage, IEEE Computer Applications in Power, pp. 26-30, vol. 10, January 1997. 
[12] K. Tomsovic, D. Bakken, V. Venkatasubramanian, A. Bose, Designing the Next Gen-eration of Actual-Time Control, Communication and Calculations for Large Power Systems, IEEE Proc. Special Issue on Energy Infrastructure Systems, Proceedings of IEEE, vol. 93, no. 5, May 2005

[13] C. Rehtanz, J. Bertsch, Wide Area Measurement and Protection System for Emergency Voltage Stability Control,Power Engineering Society Winter Meeting, 2002.IEEE, vol. 2, pp. 842 847, Jan 2002.

[14] Taylor, C.W., The Future in On-Line Security Assessment and Wide-Area Stability control, Power Engineering Society Winter Meeting, 2000. IEEE, vol. 1, pp. 78 - 83 Jan 2000.

[15] E. Nobile, A. Bose, and K. Tomsovic, Feasibility of a bilateral market for load fol-lowing, IEEE Trans. Power System. vol. 16, no.4, pp. 782787, Nov 2001.

[16] X. Yu and K. Tomsovic, Application of linear matrix inequalities for load frequency control with communication delays, IEEE Trans. Power System, vol. 19, no.3, pp. 15081515, Aug 2004.

[17] S. Bhowmik, K. Tomsovic, and A. Bose, Communication models for third party load frequency control, IEEE Trans. Power System, vol.19, no. 1, pp. 543548, Feb 2004.

[18] D. Thukaram, Transient stability analysis program, Dept. of Electrical Engineering, IISc Bangalore.

[19] Arun G. Phadke, J S Thorp Coordinated Phasor Measurements and Their Appli-cationsrm

\section{BIOGRAPHY}

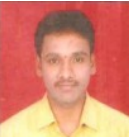

Rajesh Kumar Samala has done his B.Tech from VCE in Hyderabad in Electrical \& Electronic Engineering and has completed his M.Tech in Power Engineering \& Energy Systems. Presently he is an Assistant. Professor in EEE Dept. in NNRESGI Hyderabad. His area of interest Power Generation Control Systems

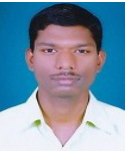

A Santosh Kumar received his B.Tech degree from Sri Indu College of Engineering and Technology in the year 2007, and he is pursuing his Masters in Electrical Power Engineering from Sreenidhi Institute of Science and Technology Hyderabad. His areas of interests include Power System Protection, Power Quality and Artificial Intelligences.

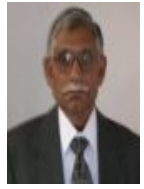

Dr. V. Kamaraju Principal at Jawaharlal Nehru Technological University, Kakinada, Professor (Visiting) at Middle East Technical University, Gaziantep, TURKEY 